\title{
Tyrosine kinase 4 is involved in the reproduction of the platyhelminth parasite Schistosoma japonicum
}

Han Ding ${ }^{1,2}$, Fengchun Liu $u^{1,2}$, Lulu Zhu ${ }^{1,2}$, Fei Wu $u^{1,2}$, Quan Liu ${ }^{1,2}$, Siyu He $e^{1,2}$, Wei Shao ${ }^{1,2}$, Yinan Du ${ }^{1,2}$, Cuiping Ren ${ }^{1,2}$, Jijia Shen ${ }^{1,2^{*}}$ and Miao Liu ${ }^{1,2^{*}}$

\begin{abstract}
Background: Schistosomiasis is one of the most common parasitic diseases affecting millions of humans and animals worldwide. Understanding the signal transduction pathways and the molecular basis of reproductive regulation in schistosomes is critically important for developing new strategies for preventing and treating these infections. Syk kinases regulate the proliferation, differentiation, morphogenesis, and survival of various types of cells and have been identified in invertebrates. Tyrosine kinase 4 (TK4), a member of the Syk kinase family, plays a pivotal role in gametogenesis in S. mansoni, affecting the development of the testis and ovaries in this parasite. The role of TK4, however, in the reproduction of S. japonicum is poorly understood.
\end{abstract}

Methods: Here, the complete coding sequence of TK4 gene in S. japonicum (SjTK4) was cloned and characterized. The expression of SjTK4 was analyzed at different life-cycle stages and in various tissues of S. japonicum by qPCR. Piceatannol, a Syk kinase inhibitor, was applied to S. japonicum in vitro. The piceatannol-induced morphological changes of the parasites were observed using confocal laser scanning microscopy and the alterations in important egg-shell synthesis-related genes were examined using $\mathrm{QPCR}$ analyses.

Results: SjTK4 mRNA was differentially expressed throughout the life-cycle of S. japonicum. SjTK4 mRNA was highly expressed in the ovary and testis of $S$. japonicum, with the level of gene expression significantly higher in males than in females. The expression levels of some important egg-shell synthesis related genes were higher in the piceatannol-treated groups than in the vehicle-treated control group and the number of eggs and germ cells also decreased in a concentration-dependent manner. Importantly, large pore-like structures can be found in the testis and ovaries of males and females after treating with piceatannol.

Conclusion: The results suggest that SjTK4 may play an important role in regulating gametogenesis of S. japonicum. The findings may help better understand the fundamental biology of $S$. japonicum. Moreover, the effect of S. japonicum treatment by piceatannol provides us with a new idea that inhibition of SjTK4 signaling pathway can effectively retard the development of the testis and ovaries.

Keywords: Schistosoma japonicum, Reproduction, SjTK4, Piceatannol

\footnotetext{
*Correspondence: shenjijia@hotmail.com; iammiaoliu@126.com ${ }^{1}$ Department of Microbiology and Parasitology, Anhui Provincial Laboratory of Microbiology and Parasitology, School of Basic Medical Sciences, 81\#Meishan Road, Hefei, Anhui 230032, People's Republic of China Full list of author information is available at the end of the article
} International License (http://creativecommons.org/licenses/by/4.0/), which permits unrestricted use, distribution, and reproduction in any medium, provided you give appropriate credit to the original author(s) and the source, provide a link to the Creative Commons license, and indicate if changes were made. The Creative Commons Public Domain Dedication waiver (http://creativecommons.org/publicdomain/zero/1.0/) applies to the data made available in this article, unless otherwise stated. 


\section{Background}

Schistosomiasis is a serious parasitic disease present in certain tropical regions worldwide, with a conservative estimate of more than 200 million affected people [1]. Schistosomiasis in humans is associated with three major parasites: Schistosoma haematobium, Schistosoma mansoni and Schistosoma japonicum. In China, S. japonicum is responsible for one of the most serious parasitic diseases in humans [2, 3]. Praziquantel had been effective in the treatment of schistosomiasis. Although praziquantel-resistant schistosomiasis has been reported [4], this is not a problem in current MDA treatment protocols. The important position of PZQ for the treatment of schistosomiasis, and an anticipation of extremely increased usage in future, create a fear of schistosomes becoming resistant to it globally [5]. Moreover, reliance on a single drug to treat schistosomiasis globally is not an effective approach for parasite control, so there exists a desperate need for alternatives. The schistosome life-cycle is complex, involving multiple developmental stages. After S. japonicum infects a definitive host, the female's reproductive system matures to produce eggs subsequent to the pairing between male and female worms. Females must pair with a male in order to undergo complete sexual development and the production of infectious eggs. After pairing with a male, the female reproductive structures, mainly the ovary and the vitelline glands, undergo terminal differentiation [6]. However, understanding the significance of the development and fundamental regulations in the reproductive biology of schistosomes, as well as in the pathogenic consequences of schistosomiasis, is still in its initial stage. Transmission of schistosomiasis depends on the release of eggs from the definitive host. Soluble egg antigens released by the miracidia within the eggs is the major cause of the pathology from the infection [7, 8]. Due to the importance of eggs for the life-cycle and for inducing pathogenesis, many genes related to the reproduction, including Vasa [9], Sj79 [10], and Wnt5 [11], have been studied in an effort to control reproductive development. Therefore, understanding the signal transduction pathways and the molecular basis of reproductive regulation in schistosomes is critically important for developing new strategies for preventing and treating schistosomiasis. Moreover, a better understanding of the fundamental biology of flatworms is also of broad scientific interest.

Tyrosine kinase 4 (TK4) is a member of the spleen tyrosine kinase (Syk) family [12]. The N-terminus of the Syk protein contains a pair of Src homology 2 (SH2) domains that are connected to each other by a linker and separated from the catalytic domain by another longer linker [13].

In vertebrates, Syk belongs to the Src family of nonreceptor tyrosine kinases, which associate directly with surface receptors, including B-cell receptors and Fcy receptors, and participate in a variety of signal transduction pathways [14]. Syk kinases are expressed in cells of the hematopoietic system and play specialized roles in inflammatory processes [15]. Syk kinases also play an important role in regulating the proliferation, differentiation, morphogenesis and survival of various types of cells [16-19]. Syk-related kinases have also been identified in invertebrates. A single Syk-related kinase, SHARK, is found in Drosophila melanogaster [20]. Sykrelated kinases have also been found in the polyp (Hydra vulgaris) and sponges [21]. However, Caenorhabditis elegans does not contain any Syk-related tyrosine kinases genes. A single Syk-related molecule, SmTK4, is found in S. mansoni; it plays a pivotal role in gametogenesis and affects the development of the testes and ovaries of $S$. mansoni [22]. Thus, the roles of the TK4 gene of $S$. japonicum (SjTK4) in the reproduction of this schistosome species warrant exploration.

In the present study, it has been confirmed that the SjTK4 gene was expressed in S. japonicum during all developmental stages both in females and, at a higher level, in males. In particular, the SjTK4 gene was highly expressed in male testes and female ovaries. To further explore the role of SjTK4 in S. japonicum, we used confocal laser scanning microscopy (CLSM) to examine the effects of piceatannol, a Syk kinase-specific inhibitor that has been previously investigated as a potential compound against schistosomes [22], on S. japonicum. Treatment of piceatannol led to significant morphological changes in the testes and ovaries of S. japonicum, as well as alterations in the mRNA levels of egg-shell formation-associated genes, including P14, P48, egg-shell precursor and FS800. Our results were consistent with previous findings in $S$. mansoni and further confirmed that the SjTK4 gene also plays an important role in regulating reproductive organ development in $S$. japonicum.

\section{Methods}

\section{Animals and parasites}

Female Kunming mice (6 weeks old) and New Zealand rabbits $(3 \pm 0.5 \mathrm{~kg})$ were purchased from the Experimental Animal Center of Anhui Province in Hefei, China. The mice were housed under specific pathogen-free conditions at Anhui Medical University. The rabbits were fed in individual cages at $19-29{ }^{\circ} \mathrm{C}$, with free access to food and water. Oncomelania hupensis snails infected with S. japonicum (a Chinese mainland strain) were provided by the Hunan Provincial Institute of Parasitic Diseases in China. To collect cercariae, a total of 50 Oncomelania hupensis snails infected with S. japonicum were exposed to light for $3-4 \mathrm{~h}\left(25-28{ }^{\circ} \mathrm{C}\right)$, and cercariae collected in a centrifuge tube and placed on ice for $15 \mathrm{~min}$. Cercariae were concentrated by centrifugation at 
$800 \times g$ for $5 \mathrm{~min}$. Mice $(n=20)$ were infected with $60-80$ cercariae, and rabbits $(n=3)$ with approximately 3000 cercariae. To collect $S$. japonicum eggs, liver tissue from rabbits 6 weeks post-infection was homogenized and then subjected to consecutive fractional filtration. The filtrate was centrifuged. The supernatant and tissue-containing layers were removed, leaving the egg-containing layer, which was diluted in $1.2 \%$ saline and passed through a nylon net (300 mesh per inch) [9]. Parasites were harvested at 16, 24 and 42 days after infection. Adult male and female parasites (at 24 and 42 days) were separated by gentle brushing. All parasites were subsequently washed three times with phosphate-buffered saline (PBS; pH 7.4) and cryopreserved at $-80^{\circ} \mathrm{C}$.

\section{Molecular cloning of SjTK4}

According to the de novo transcriptome assembly of $S$. japonicum from GenBank with the project accession number of PRJNA343582 [23], we obtained information about complete sequence of the SjTK4 gene. Primers were designed and synthesized by Sangon Biotech Co. Ltd. (Shanghai, China). The forward and reverse primers were 5'-ATG AAT GTG ACT AAT AAT GTG GTA GTA ACT CCA-3' and $5^{\prime}$-TTA AAA TGA TAT TCC ATC ACT ACC ACC A-3', respectively. For PCR amplification, a $50 \mu \mathrm{l}$ total reaction volume was prepared containing $0.2 \mu \mathrm{g}$ cDNA, $5 \mu \mathrm{mol}$ of each primer, $0.5 \mathrm{mmol}$ dNTPs and 2.5 U high-fidelity DNA polymerase (Thermo Scientific). After a denaturation step at $94{ }^{\circ} \mathrm{C}$ for $5 \mathrm{~min}$, thermal cycling was performed as follows: $94{ }^{\circ} \mathrm{C}$ for $45 \mathrm{~s}$, $60{ }^{\circ} \mathrm{C}$ for $1 \mathrm{~min}, 72{ }^{\circ} \mathrm{C}$ for $1 \mathrm{~min}$ for 30 cycles and a final extension at $72{ }^{\circ} \mathrm{C}$ for $8 \mathrm{~min}$. The PCR products were purified by agarose gel electrophoresis and sent for sequencing to Sangon Biotech Co. Ltd. (Shanghai, China). The sequencing result was uploaded to the National Center for Biotechnology Information (NCBI) database (GenBank accession number: KX984125).

\section{Amino acid sequence comparisons analysis and phylogenetic analysis of SjTK4}

The full-length cDNA sequence of SjTK4 was submitted to online analysis with BLASTX and ORF Finder in NCBI (http://www.ncbi.nlm.nih.gov/). Expasy (http:// web.expasy.org/compute_pi/) [24] was used to calculate molecular weight and isoelectric point (pI) of SjTK4. Multiple sequence alignments of SjTK4 with TK4s from other organisms were performed using DNAman. EMBOSS needle (global alignment) (http://www.ebi.ac.uk/ Tools/psa/emboss_needle/) [25] was used to analysis pairwise sequence alignments. InterProScan (http://www.ebi.ac.uk/interpro/search/sequence-search) [26] was used to analyze the primary domains of the SjTK4 protein. To confirm which family SjTK4 belonged to, phylogenetic analysis was performed based on the amino acid sequence similarity of the catalytic domain of TK4. A total of 23 sequences belonging to the Syk/Zap70 and Src families were selected for the bioinformatics analysis. All sequences were downloaded from the NCBI database (http:// www.ncbi.nlm.nih.gov/) and integrated into one file for further analysis. A phylogenetic tree was constructed using MEGA5.05 software [27]. The Neighbor-Joining method and Kimura 2-parameter model were used to estimate the evolutionary history for major clades. The reliability of phylogenetic tree was evaluated by the bootstrap analysis (1000 replicates). A cut-off value less than $50 \%$ will not be displayed. The final phylogenetic tree was obtained after editing using the graphic resources contained in the FigTree software and exported in PDF format.

\section{qPCR of SjTK4}

To detect SjTK4 gene expression, female and male parasites were cut into four parts: testis, non-testis, ovary and non-ovary [28]. Total RNA was extracted from the testis, non-testis, ovary, non-ovary, schistosomula (day 16), and adult female and male parasites at different stages (days 24 and 42) using TRIzol ${ }^{\circ}$ Reagent (Life Technologies, Carlsbad, CA, USA). RNA was quantified and used the same amount to construct the different cDNA using a PrimeScript RT reagent kit (Takara, Dalian, China). Real-time quantitative reverse transcription PCR (qPCR) was performed in duplicate using $1 \mu \mathrm{l}$ of cDNA from each sample and SYBR Premix Ex Taq II (Takara, Dalian, China) according to the manufacturer's instructions. All reactions were performed using an ABIPrism StepOnePlus ${ }^{\text {tx }}$ Real-Time PCR System (Foster City, CA, USA) with the following conditions: $95{ }^{\circ} \mathrm{C}$ for $10 \mathrm{~s}$, followed by 40 cycles of $15 \mathrm{~s}$ at $95{ }^{\circ} \mathrm{C}$ and $40 \mathrm{~s}$ at $60{ }^{\circ} \mathrm{C}$ followed by $15 \mathrm{~s}$ at $95^{\circ} \mathrm{C}, 1 \mathrm{~min}$ at $60^{\circ} \mathrm{C}$ and $95^{\circ}$ $\mathrm{C}$ for $15 \mathrm{~s}$, the threshold cycle $(\mathrm{Ct})$ values were determined using default threshold settings. The $\mathrm{Ct}$ values were defined as the fractional cycle number at which the fluorescence passes the fixed threshold, which were analyzed and generated directly by StepOne software. Primers for $\mathrm{qPCR}$ were designed and synthesized by Sangon Biotech Co. Ltd. The PCR primers for SjTK4 were $5^{\prime}$-CCT TGC TGA AGC ACG TAC AA-3' and 5'-GCC AAT TCA AGG ACA AGC AT-3'. The PCR primers for PSMD4 were 5'-ACT TTG AAC AGG AGA TGG CGA-3' and $5^{\prime}$-GCC TCA GGA CAA CGG AAC C-3'.

The relative quantification levels were calculated using the $2^{(-\Delta \Delta C T)}$ method [29]. The endogenous gene PSMD4 (26S proteasome non-ATPase regulatory subunit 4) maintains stable expression levels during the various developmental stages [30] and was thus selected as the internal reference control. 


\section{In vitro culture of adult schistosomes}

Adult schistosomes were harvested after perfusion and washed three times with M199 medium before being cultured in vitro with M199 (Catalogue number: 12340-030 Gibco, Grand Island, NY, USA) at $37{ }^{\circ} \mathrm{C}$ and $5 \% \mathrm{CO}_{2}$. The M199 medium was supplemented with an antibiotic/antimycotic mixture (1.25\%, Catalogue number: 15240-096 Gibco) and fetal calf serum (10\%, Catalogue number: 10099-141, Gibco). For each experiment, 15 pairs of $S$. japonicum were maintained in a 6-well plate in $3 \mathrm{ml}$ of culture medium.

\section{Piceatannol treatment and morphological analysis}

Piceatannol (3, 4, 39, 59-tetrahydroxy-trans-stilbene; Selleck Chemicals, Shanghai, China) was dissolved in dimethyl sulfoxide (DMSO). In each experimental group, 15 adult couples of $S$. japonicum were cultured in $3 \mathrm{ml}$ of medium and treated with one of three different concentrations of piceatannol (35 mM, $70 \mathrm{mM}$, or $100 \mathrm{mM})$. The medium with inhibitor was changed every $24 \mathrm{~h}$ for 7 days. During this time, the viability of parasites, worm pairing, and the number of eggs in the culture medium were recorded. For the morphological analysis, Schistosoma worm pairs were fixed in a solution of alcohol (95\%), formalin (3\%), and glacial acetic acid (2\%) (AFA) for at least $24 \mathrm{~h}$. Parasites were stained in hydrochloric acid-carmine dye (Ourchem, Shanghai, China) for $17 \mathrm{~h}$, and then destained in acidic 70\% ethanol. The parasites were dehydrated in a graded ethanol series $(70 \%, 90 \%$ and $100 \%)$. Parasites were cleared in $50 \%$ xylene diluted in ethanol and $100 \%$ xylene for 1 min each, and then mounted onto slides with neutral gum, sealed with cover glass, and laid flat to dry. The morphology of the reproductive organs of parasites was observed with a confocal laser scanning microscope (CLSM, Leica TCS SP5, Mannheim, Germany) using an emission wavelength of $488 \mathrm{~nm}$ and a scan excitation of $30 \%$. Images were captured and stored at $1024 \times 1024$ pixels.

\section{Quantitative analysis of some egg-shell synthesis related genes after piceatannol treatment}

Total RNA of $35 \mathrm{mM}$ and $70 \mathrm{mM}$ piceatannol treated $S$. japonicum were extracted as described above. The procedure for qPCR was conducted as described above using primers designed and synthesized by Sangon Biotech Co. Ltd. The PCR primers for P14 were $5^{\prime}$-GGA CAA CCT CCT CTG GTT CA-3' and 5'-TTT CTG GAG GTG AAT GAC GA-3', and for P48 were $5^{\prime}$-GAG CCC GTT ATA TGC CTC AA-3' and 5'-CCG GTC GAC ATA CTC CAG AT-3'. The PCR primers for eggshell precursor were 5'-TCA TGG CTT TGC AGA AAC TG-3' and 5'-GCC AAA TCT CAT CGT GTG AA-3', and for FS800 were 5' ${ }^{\prime}$-TGG AAA CGA AAG
TGA TG-3' and 5' -CTG GAA TTG AAA GGA CC-3'. The PCR primers for PSMD4 were identical to those described above.

\section{Statistical analysis}

Statistical analysis was performed using GraphPad Prism software (Version 6.0). All data were obtained from three independent experiments, each using triplicate samples and following the same protocol. The statistical significance of the difference between datasets was analyzed using Student's t-test or one-way analysis of variance (ANOVA) with multiple comparisons of Tukey's post-hoc test. Data are presented as means \pm SEM and were considered statistically significant for $P$-values $<0.05$.

\section{Results}

Cloning and sequence characteristics of the SjTK4 gene

An open reading frame of 3729 bp was amplified, encoding a predicted protein of 1242 amino acids with a predicted molecular weight of $137 \mathrm{kDa}$ (pI 8.37). Information about this protein has been uploaded to the NCBI database (GenBank: APD26308.1). A comparison of the corresponding sequences in the GenBank database from other species indicated that the TK4 amino acid sequence of S. japonicum has $76.9 \%$ identity and $82.7 \%$ similarity to that of S. mansoni (CAD13249.1). Using multiple sequence alignment of the amino acid sequences, we found that SjTK4 contains two $\mathrm{SH} 2$ domains and one TK domain. Specific conserved motifs within the TK domain were determined to be an ATPbinding region (Fig. 1a, b). Syk-TKs represent a distinct group of cellular TKs in addition to belonging to the large group of Src-like TKs, with the main difference being that Syk-TKs possess two $\mathrm{SH} 2$ domains, whereas Src-family members have one SH2 and one SH3 domain [12]. Within the Syk clade of sequences, the SjTK4 cluster was most closely related to the gene products encoded by other species, especially SmTK4 (Fig. 2).

\section{SjTK4 gene expression in different developmental stages and tissues of $S$. japonicum}

Using qPCR, we measured SjTK4 mRNA levels in eggs, cercariae and schistosomula and in the testis, ovary, non-testis and non-ovary tissues of adult male and female parasites on days 24 and 42. Levels of SjTK4 were normalized to those for the housekeeping gene PSMD. We found that the levels of the SjTK4 gene were differentially expressed in the various life-cycle stages and between the sexes of $S$. japonicum. The SjTK4 mRNA expression levels in adult parasites were markedly higher than those in immature parasites, with levels in adult males significantly higher than those in adult females $\left(F_{(6,14)}=538.1, P<0.0001\right)$ (Fig. 3). In both male and 


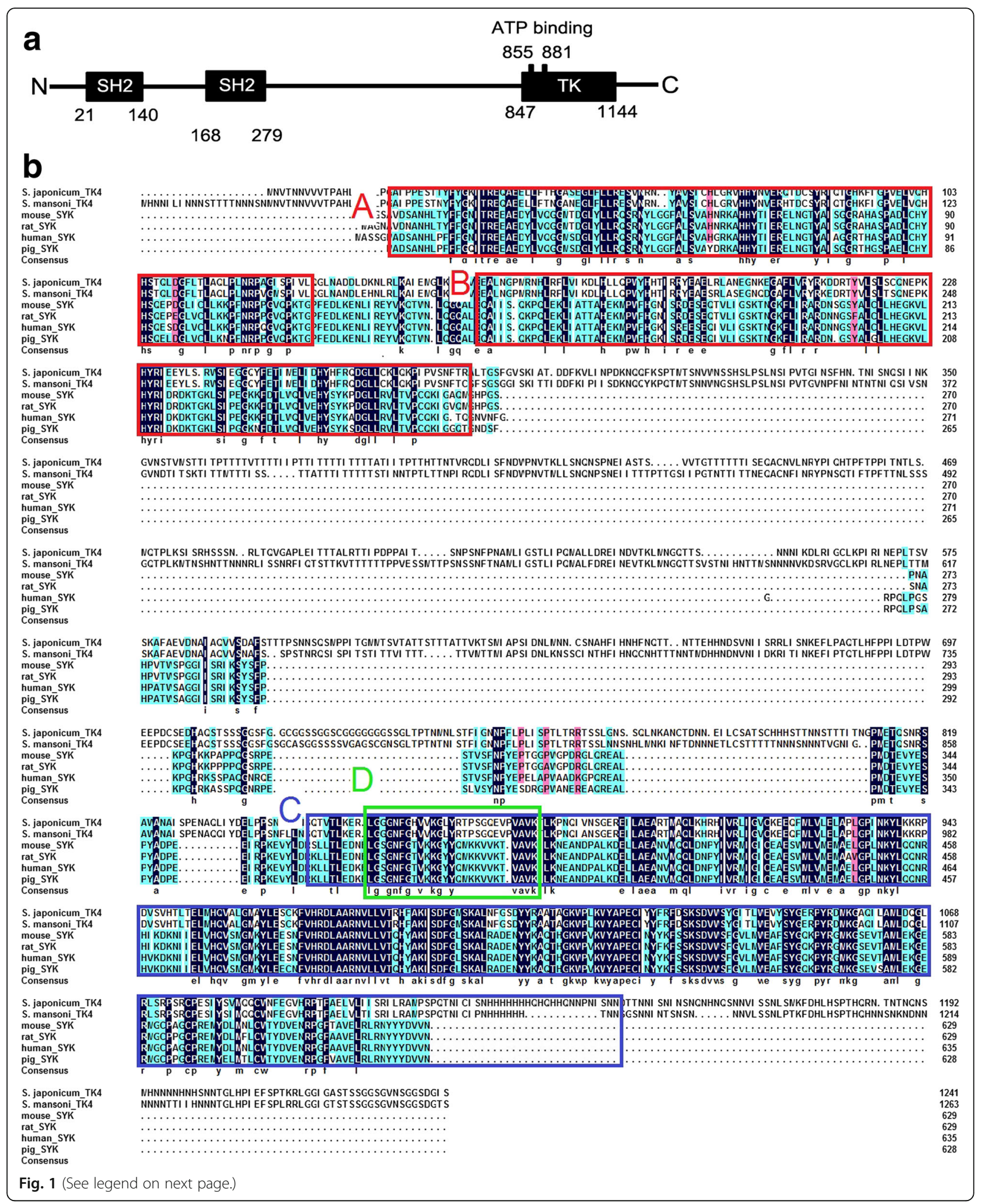


(See figure on previous page.)

Fig. 1 Schistosoma japonicum TK4 belongs to the Syk kinase family. a Structure of the TK4 domain of S. japonicum. The tandem SH2 domain is located at the N-terminus of SjTK4, whereas the catalytic tyrosine kinase domain is located at the C-terminus. They are joined together by a linker region. The numbers in the figure represent the respective sequence coordinates for SjTK4 for each domain. $\mathbf{b}$ Comparison of amino acid sequences in S. japonicum TK4 (SjTK4, APD26308.1) with other organisms: S. mansoni (SmTK4, CAD13249.1), Mus musculus (mouse Syk, AAA87462.1), Rattus norvegicus (rat Syk, NP_036890.1), Homo sapiens (human Syk, AAH01645.1), and Sus scrofa (pig Syk, NP_001098422.1). The hallmark domains are marked above the alignment, positional information refer to SjTK4. The two red boxes outline the two SH2 domains (5' SH2 position 21-140, 120aa; 3' SH2 position, 168-279, 112 aa) and the blue box outlines the TK domain (position 847-1144, 298 aa). The green box delineates a conserved the protein kinase ATP binding domain (position 855-881, 27 aa)

female parasites, higher amounts of the SjTK4 gene were expressed in the testis $\left(t_{(4)}=5.703, P=0.0047\right)$ and ovary $\left(t_{(4)}=12.96, P=0.0002\right)$ relative to non-testis and non-ovary tissues, respectively. (Fig. $4 \mathrm{a}, \mathrm{b})$.

\section{Treatment with piceatannol in vitro affect the reproduction of S. japonicum}

We found that parasite viability decreased with increasing inhibitor concentration (data not shown). To analyze the effects of piceatannol on egg production in the paired females, we counted egg numbers and found that the number of eggs $\left(F_{(2,9)}=32.59, P<0.0001\right)$ also decreased in a concentration-dependent manner compared to the DMSO-treated group (Fig. 5).

After the treatment, parasites were fixed in AFA, stained with carmine, and analyzed using CLSM. In the DMSOtreated group, no morphological anomalies were observed in the testes of the males or the ovaries of the females. The ovaries of DMSO-treated female schistosomes were composed of small immature oocytes in the anterior part and

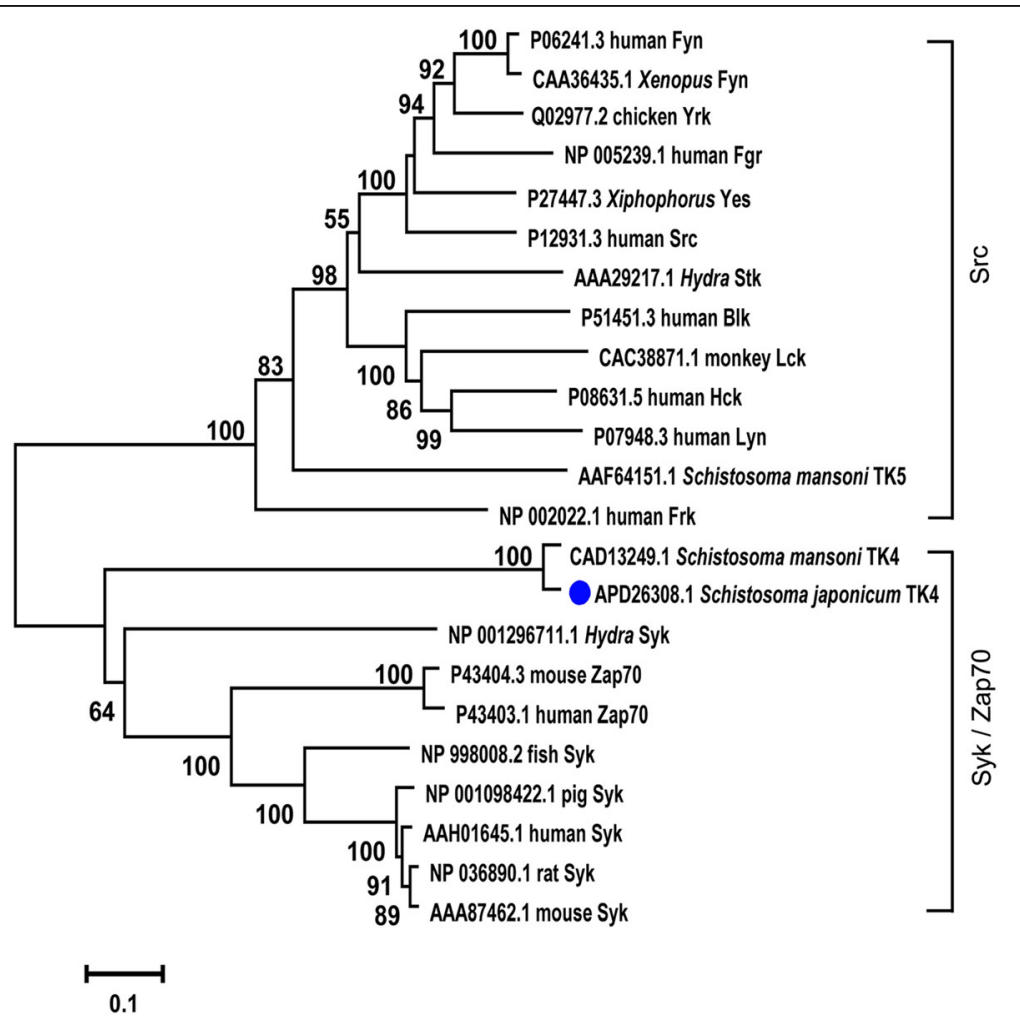

Fig. 2 Phylogenetic tree analysis of the TK4 protein in Schistosoma japonicum. All of the following sequences were obtained from the National Center for Biotechnology Information (www.ncbi.nlm.nih.gov): human Frk (NP_002022.1), Hydra Stk (AAA29217.1), human Fgr (NP_005239.1), human Src (P12931.3), Xiphophorus Yes (P27447.3), chicken Yrk (Q02977.2), human Fyn (P06241.3), Xenopus Fyn (CAA36435.1), human Blk (P51451.3), human Lyn (P07948.3), human Hck (P08631.5), monkey Lck (Saimiri sciureus; CAC38871.1), fish Syk (Danio rerio; NP_998008.2), pig Syk (NP_001098422.1), rat Syk (NP_036890.1), mouse Syk (AAA87462.1), human Syk (AAH01645.1), human Zap70 (P43403.1), mouse Zap70 (P43404.3), Schistosoma mansoni TK4 (CAD13249.1), Schistosoma mansoni TK5 (AAF64151.1), Hydra Syk (NP_001296711.1), Schistosoma japonicum TK4 (APD26308.1; indicated by the blue dot). The tree is drawn to scale, with branch lengths in the same units as those of the evolutionary distances used to infer the phylogenetic tree. The numbers above the branches indicate the bootstrap values in percentages (of 1000 replicates) 


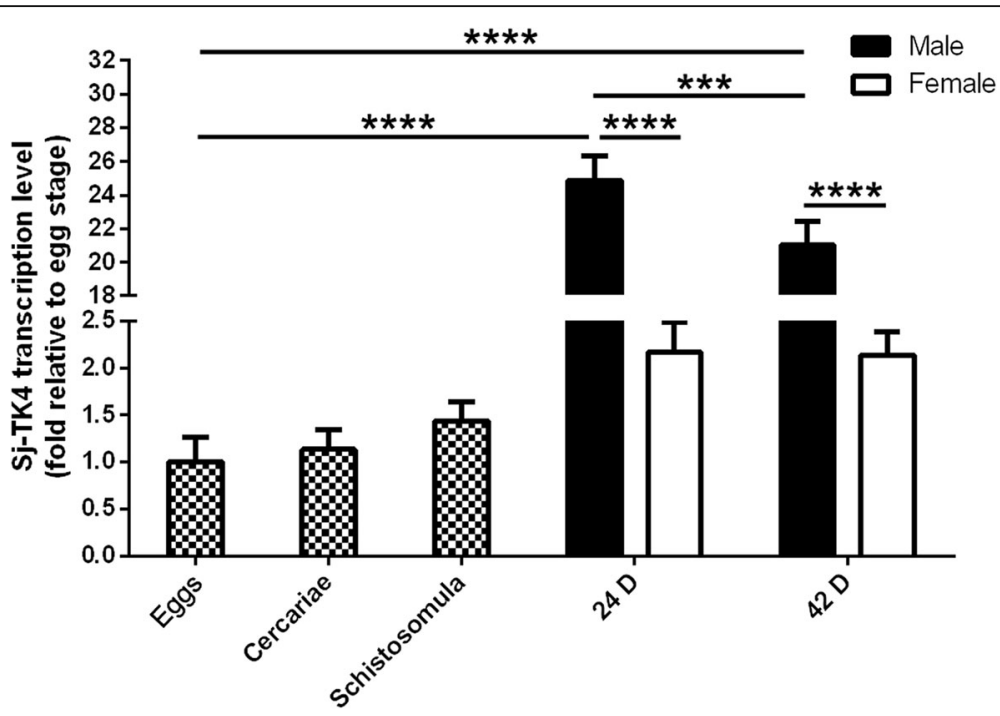

Fig. 3 Quantitative analysis of SjTK4 mRNA expression at various developmental stages and in both sexes. Data represent the mean \pm SEM of three independent experiments. Asterisks show statistical differences ( ${ }^{* * *} P<0.001{ }^{* * * *} P<0.0001$ ) tested by one-way ANOVA with multiple comparisons (Tukey's post-hoc test)

larger primary oocytes in the posterior part (Fig. 6a). The testes of DMSO-treated male schistosomes were composed of several testicular lobes arranged bead-like, and each testicular lobe contained a large number of spermatogonia at different stages and spermatocytes (Fig. 6b). In the group treated with $35 \mu \mathrm{M}$ piceatannol, the morphology of the whole germ cells in both the testis and ovary were markedly changed. In the ovaries, the sizes of the primary oocytes and immature oocytes were reduced, and the cells of the piceatannol treated groups were not as full as the cells of DMSO group (Fig. 6c). The size of the testicular lobes in the group treated with $35 \mu \mathrm{M}$ piceatannol was much smaller than that in the DMSO-treated group, and the number of spermatogonia and spermatocytes in the male testis was significantly reduced and more loosely arranged (Fig. 6d). Large pore-like structures could be found in the testis and ovaries of males and females (Fig. 6c-f, arrows). These morphological changes in both females and males were exacerbated after treatment with $70 \mu \mathrm{M}$ (Fig. 6e-f) and $100 \mu \mathrm{M}$ piceatannol (data not shown).
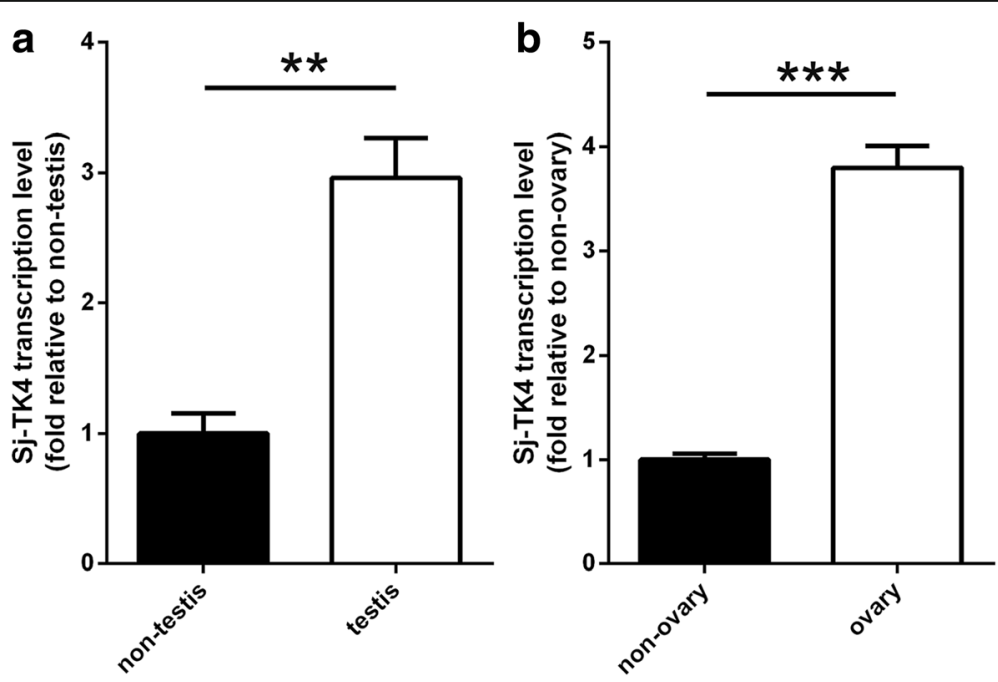

Fig. 4 Relative SjTK4 mRNA expression in the reproductive organs of Schistosoma japonicum. Quantitative analysis of ovary and non-ovary tissue in females (a) as well as testis and non-testis tissue in males (b). Data represent the mean \pm SEM of three independent experiments. Asterisks show statistical differences $\left({ }^{* *} P<0.01,{ }^{* *} P<0.001\right)$ tested by Student's t-test 


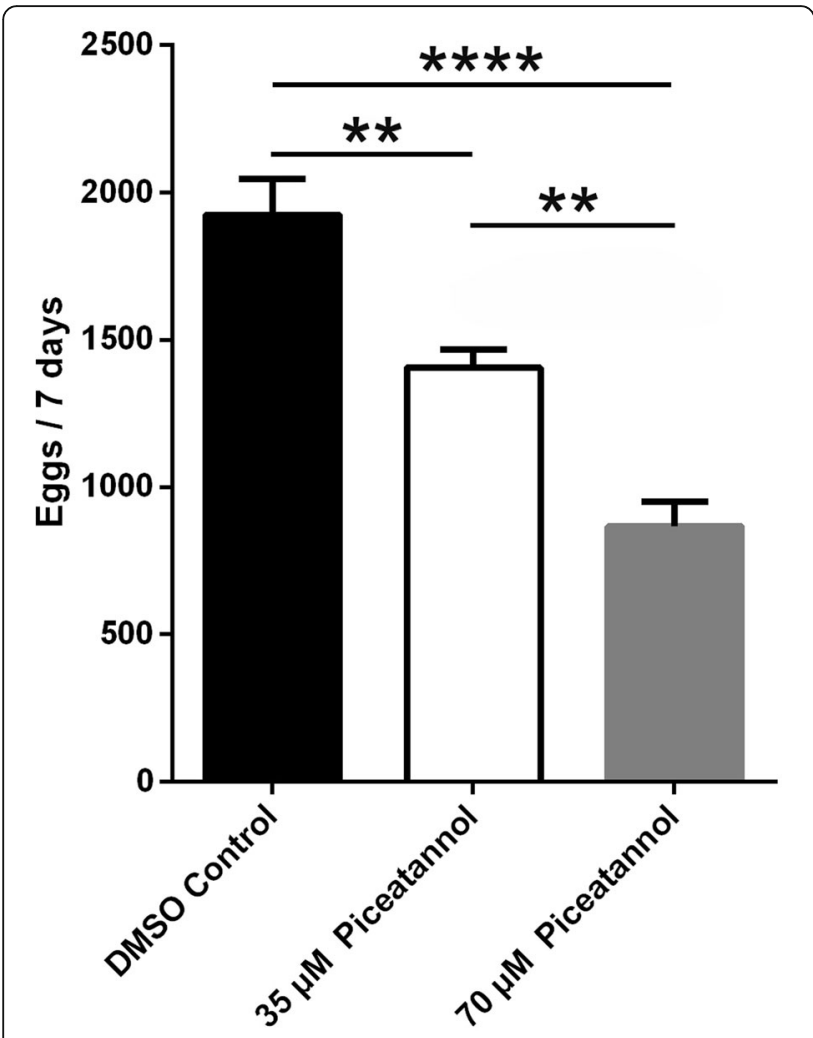

Fig. 5 Effects of piceatannol application on spawning in Schistosoma japonicum. Worm couples ( $n=15$ couples) were cultured in the absence (DMSO, control) or presence of $35 \mu \mathrm{M}$ or $70 \mu \mathrm{M}$ piceatannol. After being cultured in vitro for 7 days, the number of eggs per group was counted. Data represent the mean \pm SEM of three independent experiments. Asterisks show statistical differences ( ${ }^{* *} P<0.01$, ${ }^{* * *} P<0.0001$ ) tested by one-way ANOVA with multiple comparisons (Tukey's post-hoc test)

\section{Expression of egg-shell synthesis-related genes}

After treating $S$. japonicum with piceatannol in vitro, we performed qPCR analysis of some important egg-shell synthesis-related genes. We found that the expression levels of all these genes, including $P 14\left(F_{(2,6)}=207.9, P<\right.$ $0.0001), P 48\left(F_{(2,6)}=9.309, P=0.0145\right)$, eggshell precursor $\left(F_{(2,6)}=5.629, P=0.042\right)$, and FS800 $\left(F_{(2,6)}=34.92, P\right.$ $=0.005)$, were higher in the piceatannol-treated groups than those in the DMSO-treated group (Fig. 7a-d).

\section{Discussion}

There are several kinds of schistosomes that can infect humans. Although they have a similar life-cycle in their use of mammalian final hosts, these species differ from each other in several respects, such as time of infection and oviposition, the shape and size of eggs, and location of intravascular habitat in mammalian final hosts. These differences between species are important determinants of clinical presentation [31]. It has been confirmed that SmTK4 plays a pivotal role in gametogenesis and affects the development of the testes and ovaries of S. mansoni
[22]. It is necessary for us to confirm the findings from $S$. mansoni in S. japonicum. In the present study, we cloned and sequenced SjTK4. Our comparison of the amino acid sequences showed that SjTK4 shares $76.9 \%$ identity and $82.7 \%$ similarity with SmTK4. Therefore, it is possible that the function of SjTK4 is similar to that of SmTK4. Although significant progress has been made in the study of SmTK4 [22], further exploration of SjTK4 will help us to discover the commonalities between schistosome species. In general, small molecule kinase inhibitors that bind to kinases are broadly categorized into four major classes based on their binding mode, type I, type II, type III and type IV inhibitors [3234]. We found that human Syk (accession number AAH01645.1) shares $21.3 \%$ amino acid identity with SjTK4. The 78.7\% amino acid differences between human Syk and SjTK4 may provide a target for selective inhibitors design. However, the sequence similarity within the catalytic domain between SjTK4 and human Syk is $72.9 \%$ and the similarity of these two sequences outside the catalytic domain is only $17.2 \%$. This may be difficult for finding novel type I and type II inhibitors, but there is still hope for finding inhibitors of type III and type IV.

The SjTK4 gene was differentially expressed in the life-cycle stages and sexes of $S$. japonicum. The transcription levels of the SjTK4 gene in 24 day old male parasites were higher than those at any other stage; this coincides with the time at which paired female schistosomes start laying eggs [35]. The transcription levels of the TK4 gene in the testis and ovary were higher than those in any other tissue examined; the localization of expression of SjTK4 is consistent with that found in $S$. mansoni [22]. Therefore, we concluded that the SjTK4 signaling pathway may be important for reproductive system maturation and female oviposition after pairing with the male. The higher level of SjTK4 gene expression that we found in males, compared to females, may be because male parasites possess a greater amount of parenchymatous tissue [12].

In vertebrates and invertebrates, the differentiation of germ cells depends on the reorganization of actin and tubulin cytoskeletons [36-38]. Syk kinases are known to phosphorylate substrates involved in cytoskeleton organization or the components of the cytoskeleton, such as microtubules, directly $[19,39,40]$. In oocytes of marine nemertean worms (Cerebratulus spp.), inhibitor studies also showed that Syk kinases play a significant role during oocyte maturation. Using the Syk-kinase specific inhibitor Piceatannol (50-100 mM), a decrease in the maturation of oocytes was observed, indicated by reduced GVBD occurring in early meiosis, and also by reduced MAPK activity [41]. In our present study, after treating $S$. japonicum with piceatannol in vitro, the 

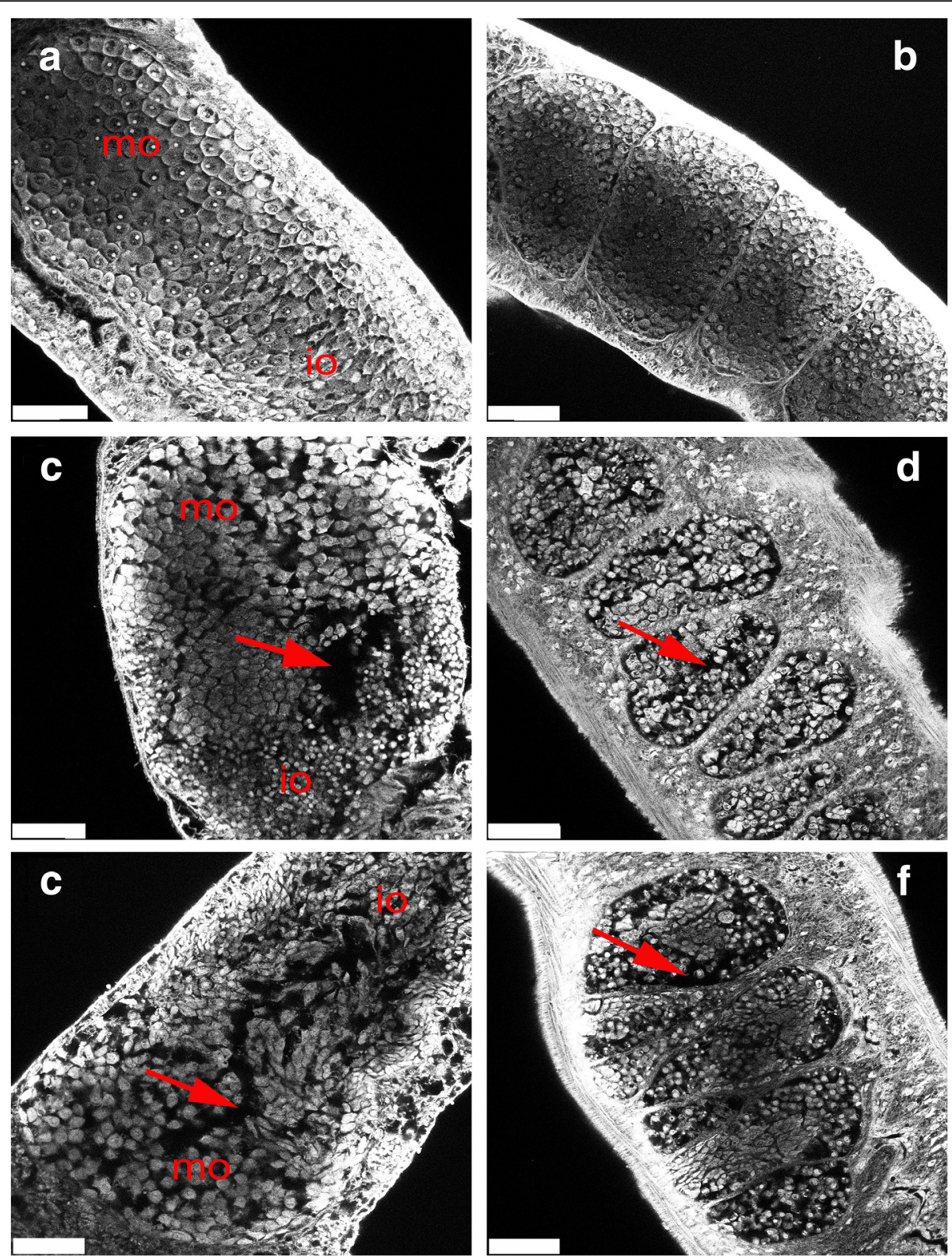

Fig. 6 Morphological changes in the testis and ovary of Schistosoma japonicum treated with piceatannol. Confocal scanning laser microscope images of carmine-stained whole-mount preparations of S. japonicum couples (a, c, e: females; $\mathbf{b}, \mathbf{d}$, $\mathbf{f}$ : males). $\mathbf{a}$, $\mathbf{b}$ Control parasites treated with DMSO only. c, d Parasites treated with $35 \mu \mathrm{M}$ piceatannol for 7 days. e, f Parasites treated with $70 \mu \mathrm{M}$ piceatannol for 7 days. Abbreviations: io, immature oocytes; mo, mature oocytes; arrows, pore-like structures. Scale-bars: $25 \mu \mathrm{m}$

number of spermatogonia and spermatocytes in the male testis were significantly reduced, and the testicular lobes were significantly smaller than those in the DMSOtreated group. We found large pore-like structures in most of the testis and ovaries of the piceatannol-treated parasites. These results indicate that piceatannol specifically inhibits the proliferation of spermatogonia in males and of primary spermatocytes in females. The proliferation of these cells is essential for the initiation and continuous production of mature germ cells [42]. A reduction in mature germ cells will inevitably reduce the number of eggs. Consequently, the differentiation of the testis and ovaries can have a significant impact on the production and development of eggs. In order to analyze the influence, the number of eggs of paired females was determined after treatment by piceatannol. We found the number of eggs also decreased in a concentrationdependent manner compared to the DMSO-treated group. This suggests that inhibition of SjTK4 signaling 

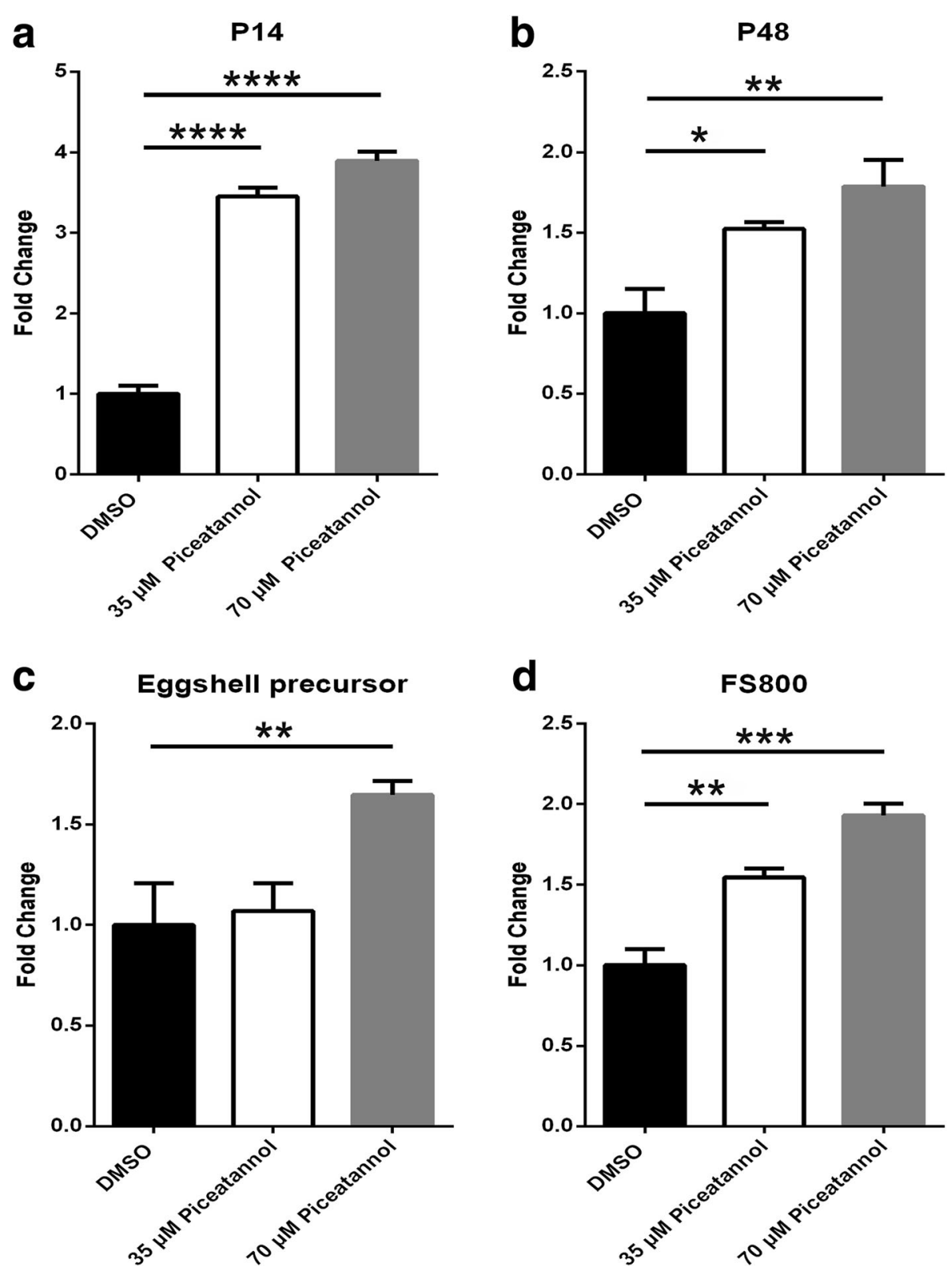

Fig. 7 Results of qPCR analyses in Schistosoma japonicum treated for 7 days with DMSO or piceatannol. Genes associated with egg-shell synthesis were investigated, including P14 (a), P48 (b), egg-shell precursor (c), and FS800 (d). Data represent the mean \pm SEM of three independent experiments. Asterisks show statistical differences $\left({ }^{*} P<0.05,{ }^{* *} P<0.01,{ }^{* * *} P<0.001\right.$, $\left.{ }^{* * *} P<0.0001\right)$ tested by one-way ANOVA with multiple comparisons (Tukey's post-hoc test)

pathway may be the alternative for blocking transmission and progression of schistosomiasis.

Many cytoplasmic tyrosine kinases have been identified in S. mansoni, such as SmTK3 (Src-like), SmTK4 (Syk-like), SmTK5, SmAbl1/2 and SmTK6 (Src/Abl-like). SmTK3, SmTK4 and SmTK6 form a trimeric kinase complex, which interacts with the receptor tyrosine kinase SmVKR1 to regulate downstream targets [43]. The Src-specific inhibitor Herb A and a TGF $\beta$ receptor inhibitor (TRIKI) can block mitosis and egg production in $S$. mansoni and downregulate egg-shell synthesis genes, including P14, FS800-like, a predicted egg-shell precursor protein gene, and tyrosinase 1 [44]. The activity of SmAbl1 is blocked by imatinib (a known Abl-TK inhibitor), which results in reduced egg production, although, unexpectedly, some genes involved in egg-shell synthesis are upregulated [45]. After treating S. japonicum with piceatannol in vitro, we found that the levels of genes involved in egg-shell production, including $P 14$, P48, egg-shell precursor and FS800, were increased, 
which was consistent with the results in imatinib-treated $S$. mansoni [45]. Many genes are involved in the complex process of egg-shell formation. The levels of unknown genes that play a critical role in this process may be decreased by piceatannol, whereas the levels of some known genes may be increased to compensate for overall reduced egg formation in piceatannol-treated schistosomes. Schistosome egg production is a complex process that involves not only the participation of different cell types, but also comprises the participation of different organs $[35,46]$. Therefore, further study is warranted to determine the molecular mechanisms whereby piceatannol affects egg formation.

\section{Conclusions}

In summary, we investigated the expression profile and provided a preliminary characterization of the TK4 gene in S. japonicum. The results of piceatannol treatment in $S$. japonicum suggest that SjTK4 may exert a regulatory function on the development of the reproductive system, supporting the hypothesis that TK4 inhibitors are potential chemotherapeutics against schistosomiasis. This study provides a good foundation for the design of more specific anti-SjTK4 drugs applicable to anti-schistosome chemotherapy.

\section{Abbreviations}

ANOVA: one-way analysis of variance; CLSM: confocal laser scanning microscopy; Ct: threshold cycle; DMSO: dimethyl sulfoxide; NCBI: National Center for Biotechnology Information; PSMD4: 265 proteasome non-ATPase regulatory subunit 4; PZQ: praziquantel; qPCR: real-time quantitative polymerase chain reaction; SH2: Src homology 2; Syk: spleen tyrosine kinase; TK4: tyrosine kinase 4

\section{Acknowledgements}

We thank a native English speaker (Science/Medical English Editing for International Researchers) for modifying the manuscript.

\section{Funding}

This work was supported by the Natural Science Foundation of China (81271865), Key University Science Research Project of Anhui Province of China (KJ2016A333) and Scientific Research of BSKY from Anhui Medical University (XJ201321).

\section{Availability of data and materials}

The datasets supporting the results are included within the article. The newly generated sequence was deposited in the GenBank database under the accession number KX984125.

\section{Authors' contributions}

$H D, M L$ and JJS conceived and designed the trial; HD, FCL, LLZ, FW, QL, SYH and WS performed the experiments; $H D, Y N D, C P R$, and $M L$ analyzed the data; ML drafted the first draft of the manuscript. JJS critically revised the manuscript. All authors read and approved the final manuscript.

\section{Ethics approval and consent to participate}

Mice received care that complied with the guidelines for the Care and Use of Laboratory Animals. All of the work presented here was approved by the Anhui Experimental Animal Training Base. The reference number is LLSC20140060. The protocols for all animal experiments were approved by the Institutional Animal Care and Use Committee at Anhui Medical University.
Consent for publication

Not applicable.

\section{Competing interests}

The authors declare that they have no competing interests. Sponsors had no role in study design, or collection, analysis and interpretation of data.

\section{Publisher's Note}

Springer Nature remains neutral with regard to jurisdictional claims in published maps and institutional affiliations.

\section{Author details}

${ }^{1}$ Department of Microbiology and Parasitology, Anhui Provincial Laboratory of Microbiology and Parasitology, School of Basic Medical Sciences, 81\#Meishan Road, Hefei, Anhui 230032, People's Republic of China. ${ }^{2}$ Anhui Key Laboratory of Zoonoses, Anhui Medical University, 81\#Meishan Road, Hefei, Anhui 230032, People's Republic of China.

Received: 9 March 2017 Accepted: 5 October 2017

Published online: 18 October 2017

\section{References}

1. Colley DG, Bustinduy AL, Secor WE, King CH. Human schistosomiasis. Lancet. 2014;383(9936):2253-64

2. Zhou XN, Wang LY, Chen MG, Wu XH, Jiang QW, Chen XY, et al. The public health significance and control of schistosomiasis in China - then and now. Acta Trop 2005;96(2-3):97-105

3. Zhou YB, Liang S, Jiang QW. Factors impacting on progress towards elimination of transmission of schistosomiasis japonica in China. Parasit Vectors. 2012;5:275.

4. Pinto-Almeida A, Mendes T, de Oliveira RN, Correa Sde A, Allegretti SM, Belo $\mathrm{S}$, et al. Morphological characteristics of Schistosoma mansoni PZQ-resistant and -susceptible strains are different in presence of praziquantel. Front Microbiol. 2016;7:594.

5. Doenhoff MJ, Cioli D, Utzinger J. Praziquantel: mechanisms of action, resistance and new derivatives for schistosomiasis. Curr Opin Infect Dis. 2008;21(6):659-67.

6. Cogswell AA, Kommer VP, Williams DL. Transcriptional analysis of a unique set of genes involved in Schistosoma mansoni female reproductive biology. PLoS Negl Trop Dis. 2012;6(11):e1907.

7. Wilson MS, Mentink-Kane MM, Pesce JT, Ramalingam TR, Thompson R, Wynn TA. Immunopathology of schistosomiasis. Immunol Cell Biol. 2007; 85(2):148-54.

8. Nady S, Shata MT, Mohey MA, El-Shorbagy A. Protective role of IL-22 against Schistosoma mansoni soluble egg antigen-induced granuloma in vitro. Parasite Immunol. 2017:39(1):pim.12392.

9. Diao Y, Hua M, Shao Y, Huang W, Liu M, Ren C, et al. Preliminary characterization and expression of vasa-like gene in Schistosoma japonicum. Parasitol Res. 2015;114(7):2679-87.

10. Jiang $Y Y, Y$ Yuan $Z Y, X u Y X$, Zang W, Cao JP, Wang $Y$, et al. [Molecular characteristics and RNA interference efficiency of Schistosoma japonicum Sj79 gene.] Zhongguo xue xi chong bing fang zhi za zhi. 2015;27(3):273-6 (In Chinese).

11. Ta N, Feng XG, Deng $L L, Z Q$ F, Hong $Y$, Liu JM, et al. Characterization and expression analysis of Wnt5 in Schistosoma japonicum at different developmental stages. Parasitol Res. 2015;114(9):3261-9.

12. Knobloch J, Winnen R. Quack M, Kunz W, Grevelding CG. A novel Syk-family tyrosine kinase from Schistosoma mansoni which is preferentially transcribed in reproductive organs. Gene. 2002;294(1-2):87-97.

13. Mocsai A, Ruland J, Tybulewicz VL. The SYK tyrosine kinase: a crucial player in diverse biological functions. Nat Rev Immunol. 2010;10(6):387-402.

14. Deng GM, Kyttaris VC, Tsokos GC. Targeting Syk in autoimmune rheumatic diseases. Front Immunol. 2016;7:78.

15. Page TH, Smolinska M, Gillespie J, Urbaniak AM, Foxwell BMJ. Tyrosine kinases and inflammatory signalling. Curr Mol Med. 2009;9(1):69-85.

16. Inatome R, Yanagi S, Takano T, Yamamura HA. Critical role for Syk in endothelial cell proliferation and migration. Biochem Biophys Res Commun. 2001;286(1):195-9. 
17. Fluck M, Zurcher G, Andres AC, Ziemiecki A. Molecular characterization of the murine Syk protein tyrosine kinase CDNA, transcripts and protein. Biochem Biophys Res Commun. 1995;213(1):273-81.

18. Tsujimura T, Yanagi S, Inatome R, Takano T, Ishihara I, Mitsui N, et al. Syk protein-tyrosine kinase is involved in neuron-like differentiation of embryonal carcinoma P19 cells. FEBS Lett. 2001;489(2-3):129-33.

19. Coopman PJ, Mueller SC. The Syk tyrosine kinase: a new negative regulator in tumor growth and progression. Cancer Lett. 2006;241(2):159-73.

20. Ferrante AW Jr, Reinke R, Stanley ER. Shark, a Src homology 2, ankyrin repeat, tyrosine kinase, is expressed on the apical surfaces of ectodermal epithelia. Proc Natl Acad Sci USA. 1995;92(6):1911-5.

21. Steele RE, Stover NA, Sakaguchi M. Appearance and disappearance of Syk family protein-tyrosine kinase genes during metazoan evolution. Gene. 1999;239(1):91-7.

22. Beckmann S, Buro C, Dissous C, Hirzmann J, Grevelding CG. The Syk kinase SmTK4 of Schistosoma mansoni is involved in the regulation of spermatogenesis and oogenesis. PLoS Pathog. 2010;6(2):e1000769.

23. Elisabeth G, Alexandre G, Christine H, Ivan I, Ron DA, Amos B. ExPASy: the proteomics server for in-depth protein knowledge and analysis. Nucleic Acids Res. 2003;31(13):3784-8.

24. Rice P1, Longden I, Bleasby A. EMBOSS: the European molecular biology open software suite. Trends Genet. 2000;16(6):276-7.

25. Quevillon E, Silventoinen V, Pillai S, Harte N, Mulder N, Apweiler R, Lopez R. InterProScan: protein domains identifier. Nucleic Acids Res. 2005;33:116-20.

26. Tamura K, Peterson D, Peterson N, Stecher G, Nei M, Kumar S. MEGA: Molecular Evolutionary Genetics Analysis using maximum likelihood, evolutionary distance, and maximum parsimony methods. Mol Biol Evol. 2011;28(10):2731-9.

27. Wang J, Yu Y, Shen H, Qing T, Zheng Y, Li Q, et al. Dynamic transcriptomes identify biogenic amines and insect-like hormonal regulation for mediating reproduction in Schistosoma japonicum. Nat Commun. 2017;8:14693.

28. Zhu L, Zhao J, Wang J, Hu C, Peng J, Luo R, et al. MicroRNAs are involved in the regulation of ovary development in the pathogenic blood fluke Schistosoma japonicum. PLoS Pathog. 2016;12(2):e1005423.

29. Milligan JN, Jolly ER. Identification and characterization of a Mef2 transcriptional activator in schistosome parasites. PLoS Negl Trop Dis. 2012;6(1):e1443.

30. Liu S, Cai P, Hou N, Piao X, Wang H, Hung T, et al. Genome-wide identification and characterization of a panel of house-keeping genes in Schistosoma japonicum. Mol Biochem Parasitol. 2012;182(1-2):75-82.

31. Ferrari TC, Moreira PR. Neuroschistosomiasis: clinical symptoms and pathogenesis. Lancet Neurol. 2011;10(9):853-64.

32. Gavrin LK, Saiah E. Approaches to discover non-ATP site kinase inhibitors. Medchemcomm. 2013;4(1):41-51.

33. Simard JR, Kluter S, Grutter C, Getlik M, Rabiller M, Rode HB, et al. A new screening assay for allosteric inhibitors of cSrc. Nat Chem Biol. 2009;5(6):394-6.

34. Ohren JF, Chen H, Pavlovsky A, Whitehead C, Zhang E, Kuffa P, et al. Structures of human MAP kinase kinase 1 (MEK1) and MEK2 describe novel noncompetitive kinase inhibition. Nat Struct Mol Biol. 2004;11(12):1192-7.

35. Kunz W. Schistosome male-female interaction: induction of germ-cell differentiation. Trends Parasitol. 2001;17(5):227-31.

36. Xiao X, Yang WX. Actin-based dynamics during spermatogenesis and its significance. J Zhejiang Univ Sci B. 2007;8(7):498-506.

37. Mansir A, Justine JL. The microtubular system and posttranslationally modified tubulin during spermatogenesis in a parasitic nematode with amoeboid and aflagellate spermatozoa. Mol Reprod Dev. 1998;49(2):150-67.

38. Sun QY, Schatten $H$. Regulation of dynamic events by microfilaments during oocyte maturation and fertilization. Reproduction. 2006;131(2):193-205.

39. Peters JD, Furlong MT, Asai DJ, Harrison ML, Geahlen RL. Syk, activated by cross-linking the B-cell antigen receptor, localizes to the cytosol where it interacts with and phosphorylates alpha-tubulin on tyrosine. J Biol Chem. 1996;271(9):4755-62.

40. Faruki S, Geahlen RL, Asai DJ. Syk-dependent phosphorylation of microtubules in activated B-lymphocytes. J Cell Sci. 2000;113(Pt 14):2557-65.

41. Stricker SA, Smythe TL. Differing mechanisms of CAMP- versus seawaterinduced oocyte maturation in marine nemertean worms II. The roles of tyrosine kinases and phosphatases. Mol Reprod Dev. 2006;73(12):1564-77.

42. Braydich-Stolle L, Kostereva N, Dym M, Hofmann MC. Role of Src family kinases and N-Myc in spermatogonial stem cell proliferation. Dev Biol. 2007; 304(1):34-45.
43. Beckmann S, Hahnel S, Cailliau K, Vanderstraete M, Browaeys E, Dissous C, et al. Characterization of the Src/Abl hybrid kinase SmTK6 of Schistosoma mansoni. J Biol Chem. 2011;286(49):42325-36.

44. Buro C, Oliveira KC, Lu Z, Leutner S, Beckmann S, Dissous C, et al. Transcriptome analyses of inhibitor-treated schistosome females provide evidence for cooperating Src-kinase and TGFbeta receptor pathways controlling mitosis and egg-shell formation. PLoS Pathog. 2013;9(6):e1003448,

45. Buro C, Beckmann S, Oliveira KC, Dissous C, Cailliau K, Marhofer RJ, et al. Imatinib treatment causes substantial transcriptional changes in adult Schistosoma mansoni in vitro exhibiting pleiotropic effects. PLoS Negl Trop Dis. 2014;8(6):e2923.

46. Popiel I, Basch PF. Reproductive development of female Schistosoma mansoni (Digenea: Schistosomatidae) following bisexual pairing of worms and worm segments. J Exp Zool. 1984;232(1):141-50.

\section{Submit your next manuscript to BioMed Central and we will help you at every step:}

- We accept pre-submission inquiries

- Our selector tool helps you to find the most relevant journal

- We provide round the clock customer support

- Convenient online submission

- Thorough peer review

- Inclusion in PubMed and all major indexing services

- Maximum visibility for your research

Submit your manuscript at www.biomedcentral.com/submit
C Biomed Central 